\title{
Interventional oncology for older patients in liver and kidney malignancies
}

\author{
"Age alone should not be considered as a risk factor in carefully selected \\ patients for interventional oncology procedures, but in the future a specific \\ score to predict the risk in older patients will be appropriate."
}

A lot of information has been accumulated in the last 20 years through retrospective and prospective clinical trials in the various solid tumors for older patients requiring medical treatment. The results provide indications useful for the management of most tumor types in elderly patients. Much has also been produced concerning the principles and practice of multidimensional geriatric assessment (MGA) in medical oncology: consensus has been achieved on the ability of MGA to identify three different categories of elderly cancer patients on the basis of their life expectancy: those in very good condition or 'fit', who may receive the same medical treatment (chemotherapy) as younger patients; those partially impaired, who require tailored approaches and moderate assistance; and the frail ones, who are candidates only for supportive care [1,2].

However, the problems of cancer surgery in the elderly have also been evaluated with increasing attention. A validated instrument such as the MGA may also predict short-term surgical outcomes more precisely. To predict the surgical risk in older patients the surgical risk assessment tools that are currently used have in fact been brought together with geriatric assessment tools such as the MGA and the Brief Fatigue Inventory (BFI) to obtain a comprehensive picture, in terms of health and functionality, of the oncogeriatric patient. This battery of validated instruments now forms the Preoperative Assessment of Cancer in the Elderly (PACE) and represents a useful tool in evaluating oncogeriatric fitness for surgery, but it has never been used in older patients before interventional oncology procedures [3,4].

While planning a loco-regional intervention for an older cancer patient we must decide whether this case should be treated with surgery, radiotherapy, or more recently, predominantly in liver and kidney cancer, with a third option, that is to say interventional oncology. Within interventional radiology in fact, techniques for the diagnosis and treatment of cancer such as transcatheter and percutaneous techniques have been adopted for solid organ pathologies with increased efficacy and reduced rates of morbidity and mortality, leading to the development of the field of interventional oncology [5].

Not much information from randomized trials is presently available on results concerning the possible advantage offered by interventional oncology in older patients; however, less invasive procedures, such as those provided by this modality, are often offered to older patients believing that the benefits outweigh the drawbacks for nonfit vulnerable older patients. Primary liver cancer and kidney neoplasia are two of the most frequent tumors in older patients [6,7].

Bland arterial embolization has been proven to be able to provide an important palliation in patients who are not eligible for surgery and as salvage therapy for patients with recurrent disease after resection for primary and metastatic liver cancer, alone or combined with ablative techniques, such as radiofrequency ablation (RFA) [8].

Thornton et al. are the only authors who have been studying the problem of treating with bland arterial embolization hepatocarcinoma in patients older than 70 years [9]. The mean age of their patient population was in fact 75 years. No significant differences between younger and older groups in the incidence of severity and mortality for infectious, hepatobiliary, renal, vascular or miscellaneous complications was observed. In the older group the 30-day mortality was 3.5\% (six out of 186) and the major complication rate $10.7 \%$. Survival was similar in the two groups, although patients older than 70 years with cardiovascular morbidities more often had cardiovascular complications. Length of hospitalization did not, however, differ in the two age groups. No report is currently available about liver RFA in older patients.

RFA in the kidney is now performed as a standard therapeutic nephron-sparing option in patients who are poor candidates for resection [10], but no report on the comparison of results of renal RFA between young and older

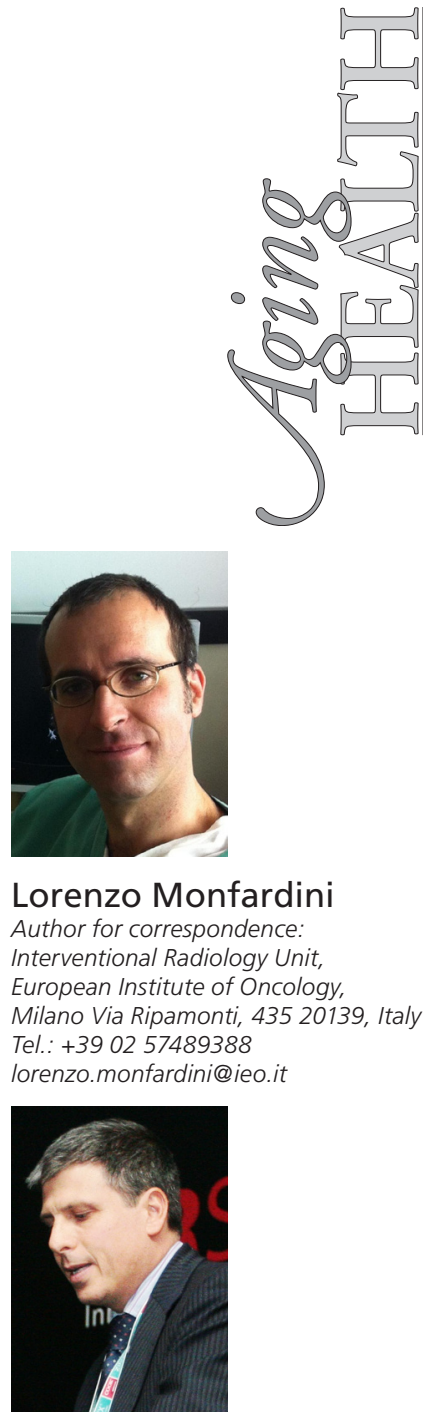

Franco Orsi

Interventional Radiology Unit, European Institute of Oncology, Milano, Italy

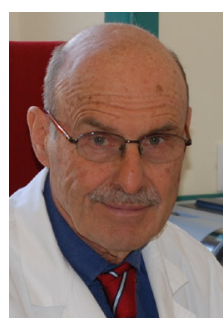

Silvio Monfardini

Geriatric Oncology Program.

Fondazione Don Gnocchi, Milano, Italy

Keywords

- bland arterial embolization

- interventional oncology • older

patients $\bullet$ radiofrequency ablation Future 
patients is available. However, the procedure is currently also used in older patients. During the early experiences with RFA, one study reported on the pooled experience from 133 RFA from four institutions $1.8 \%$ major and $9.2 \%$ minor complications [11]. The complication rate has diminished with more RFA experience. Since there are no randomized controlled trials comparing the effects of RFA with the standard surgical treatment, RFA cannot yet be considered an established procedure.

But for patients in whom nephrectomy has been excluded (high risk of renal failure or relapse after surgery) as an alternative to surgery, RFA is increasingly applied. This procedure compared to nephron-sparing surgery allows the avoidance of transient renal artery clamping and overall risk of renal ischemia [12]. This can then definitely be useful for elderly patients with renal comorbidity or simply age-related impaired renal function.

In conclusion, a choice between hepatectomy rather than bland arterial embolization or RFA for liver malignacies is difficult since the relative indications concern different stages of the disease. But in older patients, even those with associated comorbidities, who are not amenable to the surgical intervention, interventional oncology could provide a consistent palliation.

Concerning kidney carcinoma, while for large kidney mass the indication to perform locoregional treatments such as RFA instead of surgery is still controversial, in patients of older age and with smaller tumor size they are presently performed more frequently. Probably in older patients, although still not proven by controlled randomized studies, RFA is already believed to offer a shorter hospital-stay with lower comorbidities and could be more easily offered than surgery to vulnerable and frail patients.

The hope for the future is that before the interventional oncology procedures a MGA will be performed. Thus it will be possible to determine whether vulnerable or unfit patients who are not self-sufficient with kidney carcinoma, or even with potentially resectable liver neoplastic disease, could also be considered as possible candidates for interventional oncology techniques instead of surgery.

\section{Conclusion}

Interventional oncology may play a key role in multidisciplinary management of older patients with liver and kidney malignancies; arterial liver bland embolization even followed by radiofrequency ablation and renal radiofrequency ablation are safe and effective in older patients. Age alone should not be considered as a risk factor in carefully selected patients for interventional oncology procedures, but in the future a specific score to predict the risk in older patients will be appropriate.

\section{Financial \& competing interests disclosure}

The authors have no relevant affiliations or financial involvement with any organization or entity with a financial interest in or financial conflict with the subject matter or materials discussed in the manuscript. This includes employment, consultancies, honoraria, stock ownership or options, expert testimony, grants or patents received or pending, or royalties.

No writing assistance was utilized in the production of this manuscript.

\section{References}

1. Balducci L, Yates J. General guidelines for the management of older patients with cancer. Oncology 14(11A), 221-227 (2000).

2. Extermann M, Aapro M, Bernabei R et al. Task force on CGA of the International Society of Geriatric Oncology use of comprehensive geriatric assessment in older cancer patients: recommendations from the task force on CGA of the International Society of Geriatric Oncology (SIOG). Crit. Rev. Oncol. Hematol. 55, 241-252 (2005).

3. Audisio RA, Gennari R, Sunouchi K et al. Preoperative assessment of cancer in elderly patients: a pilot study. Support Cancer Ther. 1, 155-160 (2003).

4. Kristjansson SR, Farinella E, Gaskell S, Audisio RA. Surgical risk and post-operative complications in older unfit cancer patients.
Cancer Treat. Rev. 35, 499-502 (2009).

5. Goldberg SN, Bonn J, Dodd G et al. Society of Interventional Radiology Interventional Oncology Task Force: interventional oncology research vision statement and critical assessment of the state of research affairs. J. Vasc. Interv. Radiol. 10, 1287-1294 (2005).

6. Gomaa AI, Shahid AK, Toledano MB, Waked I, Taylor-Robinson SD. Hepatocellular carcinoma: epidemiology, risk factors and pathogenesis. World J. Gastroenterol. 14, 4300-4308 (2008)

7. McLaughlin, Lipwort L, Tarone RE et al. Renal cancer: in prevention. In: Cancer Epidemiology and Prevention. Schottenfeld D, Fraumeni J (Eds). Oxford University Press, NY, USA, 1087-1100 (2006).

8. Liapi E, Geschwind JF. Transcatheter and ablative therapeutic approaches for solid malignancies. J. Clin. Oncol. 25(8), 978-986 (2007).

9. Thornton HT, Cove YA, Petre EN et al. Comparison of outcomes from treating hepatocellular carcinoma by hepatic artery embolysation in patients younger and older than 70 years. Cancer 21, 5000-5006 (2009).

10. Venkatesan AM, Wood BJ, Gervais DA. Percutaneous ablation in the kidney. Radiology 261(2), 375-91 (2011).

11. Sangtae P, Cadeddu JA. Outcomes of radiofrequency ablation for kidney cancer. Cancer Control 14, 205-210 (2007).

12. Raman JD, Raj GV, Lucas SM, Williams SK et al. Renal functional outcomes for tumours in a solitary kidney managed by ablative or extirpative techniques. BJU Int. 4, 496-500 (2010). 